\title{
Parallel PWMs Based Fully Digital Transmitter with Wide Carrier Frequency Range
}

\author{
Bo Zhou, Kun Zhang, Wenbiao Zhou, Yanjun Zhang, and Dake Liu \\ School of Information and Electronics, Beijing Institute of Technology, Beijing 100081, China \\ Correspondence should be addressed to Bo Zhou; zhoubo07@bit.edu.cn
}

Received 4 July 2013; Accepted 6 August 2013

Academic Editors: A. Garcia-Zambrana and A. Jugessur

Copyright (C) 2013 Bo Zhou et al. This is an open access article distributed under the Creative Commons Attribution License, which permits unrestricted use, distribution, and reproduction in any medium, provided the original work is properly cited.

\begin{abstract}
The carrier-frequency (CF) and intermediate-frequency (IF) pulse-width modulators (PWMs) based on delay lines are proposed, where baseband signals are conveyed by both positions and pulse widths or densities of the carrier clock. By combining IF-PWM and precorrected CF-PWM, a fully digital transmitter with unit-delay autocalibration is implemented in $180 \mathrm{~nm}$ CMOS for high reconfiguration. The proposed architecture achieves wide CF range of $2 \mathrm{M}-1 \mathrm{GHz}$, high power efficiency of $70 \%$, and low error vector magnitude (EVM) of 3\%, with spectrum purity of $20 \mathrm{~dB}$ optimized in comparison to the existing designs.
\end{abstract}

\section{Introduction}

Wireless communication is becoming more and more important and ubiquitous in modern society. To support numbers of communication standards in small and same handheld devices, there are growing demands for flexible transmitters and receivers supporting multimode communications with high efficiency. Recently, lots of researches have been conducted in RF reconfigurable transceivers using novel hardware implementation. This paper focuses on fully digital wireless transmitters.

The existing design $[1,2]$, employing all-digital phaselocked loop (ADPLL) and delta-sigma modulator, introduces large fractional spurs and requires strict energy match between power branches. The existing transmitters with quadrature $[3,4]$ or delay-line $[5,6]$ based intermediatefrequency (IF) pulse-width modulation (PWM), are not apt for low carrier-frequency (CF) applications, under which the band-pass filter (BPF) fails to suppress IF component or harmonics closer to the carrier. The existing radio-frequency (RF) PWM [7] or direct digital frequency synthesizer (DDFS) [8] based transmitters, aiming for low CF conditions, are not considered as fully digital designs, since analogue feedback or mixed-signal configurations are used. The existing architecture [9] employing outphasing amplification technique is not widely used in commerce due to strict matching requirements between dual paths and distortion and efficiency degradation caused by RF power combiner.

In this paper, delay-line based CF-PWM with precorrection logic is proposed, where the only carrier clock is employed to ensure spectrum purity under low CF conditions. By combining IF-PWM for high $\mathrm{CF}$ and precorrected CF-PWM for low CF, a fully digital transmitter is presented with wide CF range and high efficiency. A unitdelay autocalibration loop for delay lines is also proposed with reconfigurable carrier frequency $f_{C}$.

\section{Architecture}

Figure 1 shows the proposed fully digital transmitter with parallel IF- and CF-PWMs. The coordinate rotation digital computer (CORDIC) algorithm accomplishes the conversion from $I / Q$ to polar coordinate $A / \Phi[10]$. The wide CF range of $2 \mathrm{M}-1 \mathrm{GHz}$ is divided into low $f_{\mathrm{C}}$ band of $2 \mathrm{M}-100 \mathrm{MHz}$ and high $f_{C}$ band of $80 \mathrm{M}-1 \mathrm{GHz}$. About $20 \mathrm{MHz}$ frequency overlap area is chosen to avoid the switching jitter between high and low $f_{C}$ bands. Under low CF band, the transmitter working in mode " 1 " and CF-PWM enabling, multibit phase component $\Phi$ is mapped to 1/4-period position of the carrier clock, and multibit envelope component $A$ is converted to precorrected pulse width of the carrier clock. For high CF case, mode " 2 " and IF-PWM enabling, phase $\Phi$ is conveyed 


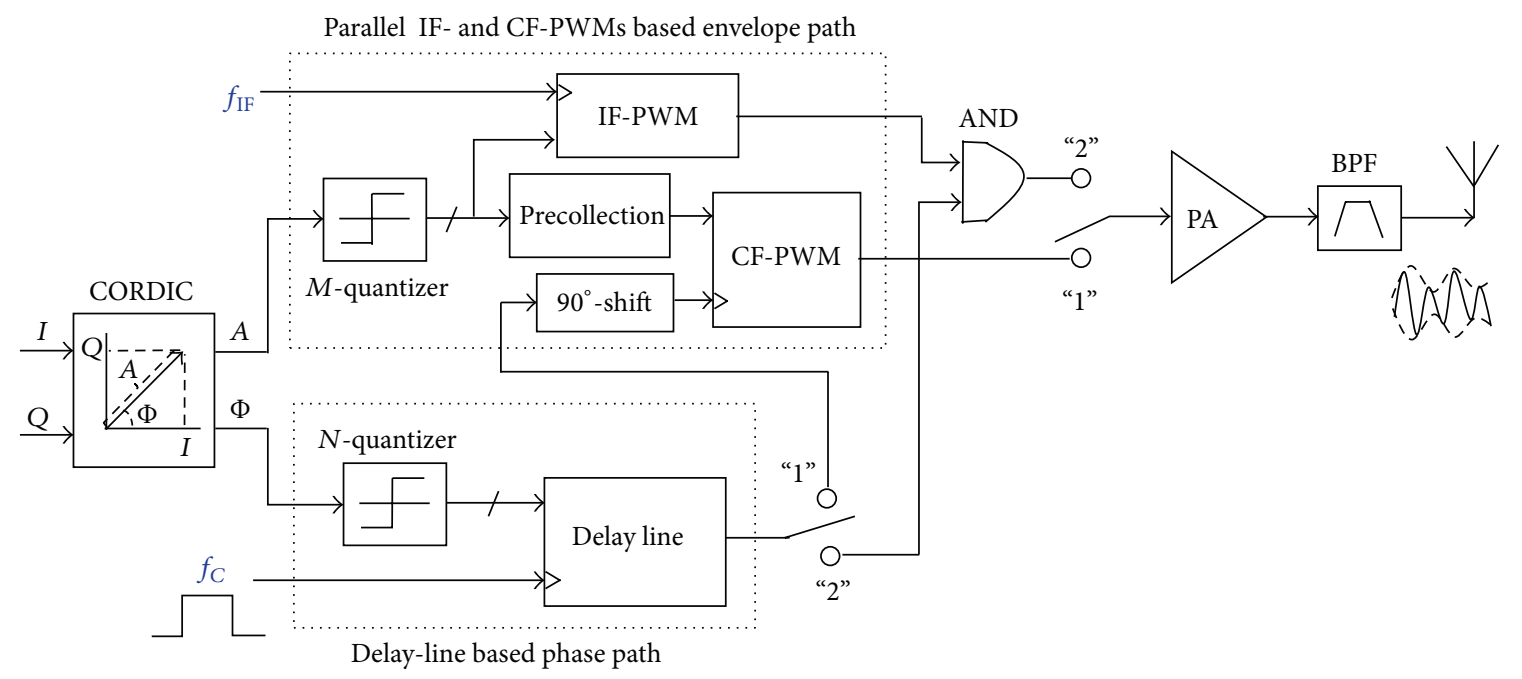

"1": low carrier frequency
"2": high carrier frequency

FIGURE 1: Proposed fully digital transmitter with parallel IF- and CF-PWMs.

by rising-edge position of the carrier clock, and envelope $A$ is represented by pulse width of an IF clock and subsequently converted to pulse density of the carrier clock. The positionand pulse-modulated carrier clock, amplified by a switchedmode class-D power amplifier (PA), is reshaped to the phaseand envelope-modulated sinusoidal carrier by an BPF with CF or IF harmonics suppressed.

The output sinusoidal amplitude $y$ and input duty cycle $d$ of the BPF conform to (1), according to Fourier series expansion of periodic square wave [7]. For IF-PWM mode, it is the pulse densities rather than pulse widths of the carrier clock that represent baseband envelopes; the BPF handling standard square waves with a fixed duty cycle of 0.5 does not encounter any nonlinear distortion. However, for CF-PWM one, the pulse widths or duty cycles on behalf of baseband envelopes are variable. That is, the BPF not only introduces envelope distortions, but also degrades original rising-edge phases hidden in the modulated carrier due to zero-crossing point deviations. Therefore, to ensure the modulation linearity of CF-PWM mode, an envelope precorrection module with an inverse function of (1) is added to compensate for the envelope offset, and $1 / 4$-period ( $90^{\circ}$-shifted point) positions rather than rising-edge (zero-crossing point) positions of the carrier clock are modulated to convey phase components without distortion as follows:

$$
y= \begin{cases}\frac{2}{\pi}, & \text { IF-PWM }(d=0.5), \\ \frac{2}{\pi} \sin (\pi \times d), & \text { CF-PWM. }\end{cases}
$$

For CF-PWM mode, only CF clock is involved in signal modulations, whereas IF clock or aliasing is avoided and carrier harmonics are effectively eliminated by BPF. Under IF-PWM condition with less limitation or enough margin between $f_{C}$ and signal bandwidth, although IF clock $f_{\mathrm{IF}}$ is introduced, the BPF could still suppress IF interferences by setting reasonable relations between $f_{C}, f_{\mathrm{IF}}$, and BPF bandwidth $B_{\mathrm{RF}}$, following (2)

$$
\begin{gathered}
\frac{f_{\mathrm{C}}}{B_{\mathrm{RF}}} \leq 50, \quad \frac{f_{\mathrm{IF}}}{B_{\mathrm{RF}}} \geq 2.5, \\
k+1>\frac{f_{\mathrm{C}}}{f_{\mathrm{IF}}} \neq k+0.5>k, \quad k=10 \sim 19 .
\end{gathered}
$$

With 6th order BPF, IF spurs lower than $-80 \mathrm{~dB}$ are ensured when $f_{\mathrm{IF}}$ is more than 5 times farther than the BPF corner and when the tenth-order or higher IF harmonics with less power locate around the carrier band. With normalized envelope values higher than 0.1 , there is at least one whole carrier clock transmitted to convey baseband components during each IF period. In addition, the ratio $f_{C} / f_{\mathrm{IF}}$ should not be a multiple of 0.5 to avoid IF harmonics located at $2 f_{C}$ down-converts with the carrier at $f_{C}$ into the signal band. Here, $f_{\mathrm{IF}}=f_{\mathrm{C}} / 15.4$.

\section{Implementation}

3.1. IF-PWM Mode. Figure 2 shows delay-line based IFPWM mode for the proposed transmitter. Multibit phase components are mapped to the rising-edge positions of the carrier clock with a fixed duty cycle of 0.5 by employing an $N$-level quantizer and an $N$-stage delay line where the continuous phases of $0-360^{\circ}$ are equidistantly quantized to $N$ discrete values. Multibit envelope components are converted to the pulse widths of the IF clock, which are subsequently converted by AND operation to the pulse densities of the carrier clock with the fixed pulse width inversely proportional to $f_{C}$. Under enough margins and perfect values between $f_{C}, f_{\mathrm{IF}}$, and $B_{\mathrm{RF}}$, the BPF effectively suppresses the IF spurs or harmonics. Modulation 


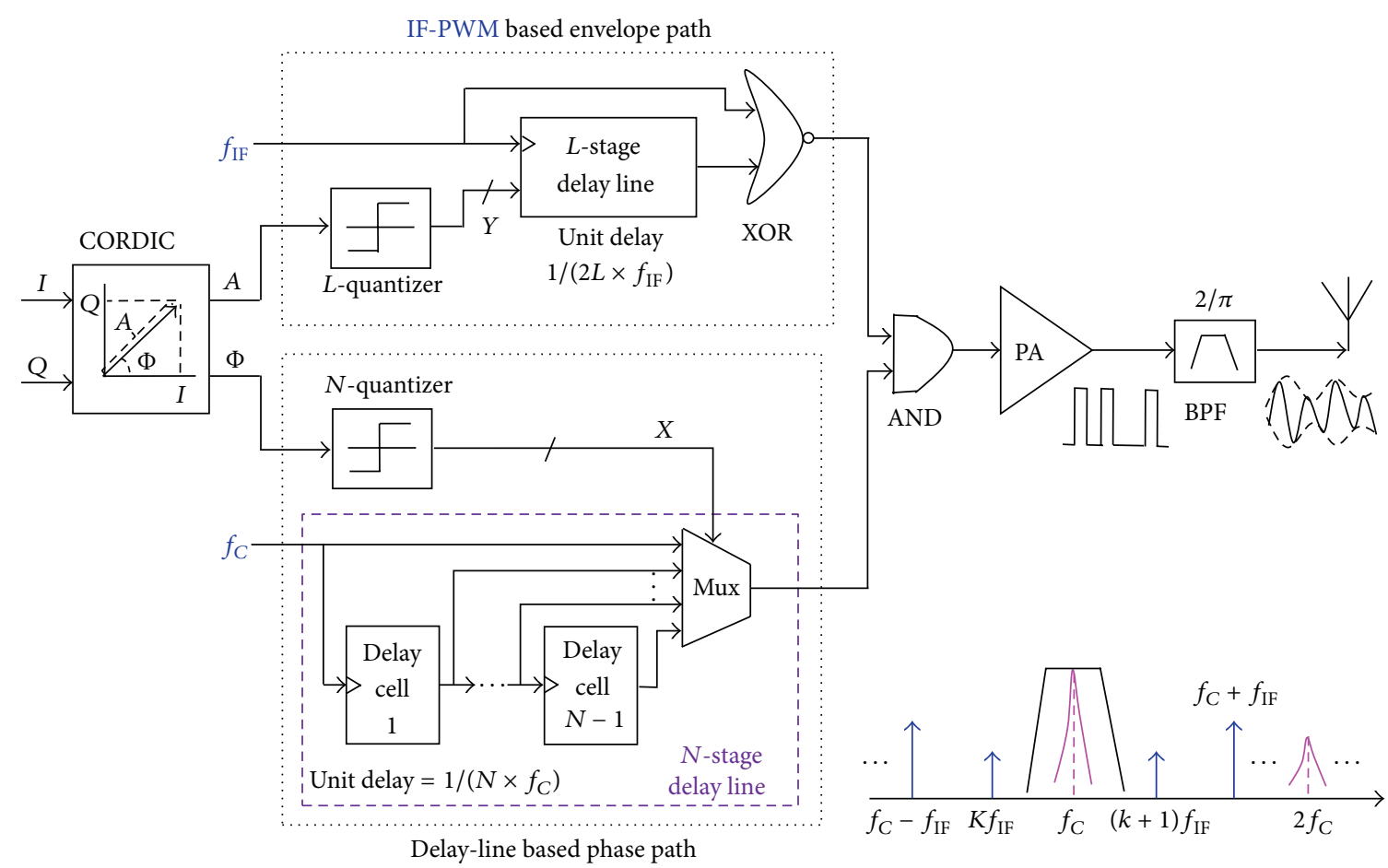

FIGURE 2: Delay-line based IF-PWM mode for proposed fully digital transmitter.

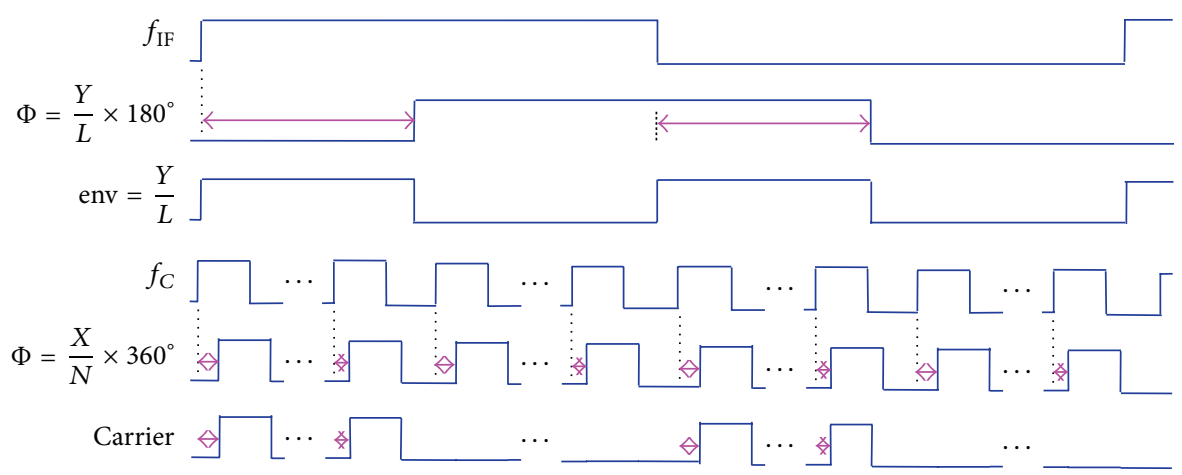

FIGURE 3: Function diagram of IF-PWM architecture.

linearity strongly depends on the quantization resolutions or levels.

The function diagram of the IF-PWM mode is shown in Figure 3 . For a certain carrier frequency $f_{C}$, the $N$-stage delay line with a unit delay of $1 /\left(N \times f_{C}\right)$ converts the quantized phase $X$ to the rising-edge lag $\Phi$ of the carrier clock. The $L$-stage delay line with a unit delay of $1 /\left(2 L \times f_{\mathrm{IF}}\right)$ maps the quantized envelope $Y$ into the rising-edge lag $\Phi$ of the IF clock. The pulse-width env generated by $\Phi$ shapes the pulse density of the carrier clock to represent the envelope component, and the rising-edge lag $\Phi$ of the carrier clock reflects the phase component, respectively.

Assuming baseband envelope $A(t)$, phase $\Phi(t)$, and envelope normalized value $A_{\text {std }}$, the modulated carrier clock $c(t)$ is shown in (3), where square() is the function of periodic square wave and $\omega_{C}$ is the carrier angular frequency. One has

$$
c(t)=\frac{A(t)}{A_{\text {std }}} \times \text { square }\left[\omega_{c} t+\Phi(t)\right] .
$$

After filtered smoothly by the sequent BPF, the modulated sinusoidal carrier $y(t)$ is shown in (4). Continuous baseband envelope $A$ and phase $\Phi$ are transmitted:

$$
\begin{aligned}
y(t) & =\frac{A(t)}{A_{\text {std }}} \times \frac{2}{\pi} \times \sin \left[\omega_{c} t+\Phi(t)\right] \\
& =\frac{2}{\pi \times A_{\text {std }}}\left[A(t) \times \sin \left(\omega_{c} t+\Phi(t)\right)\right] .
\end{aligned}
$$




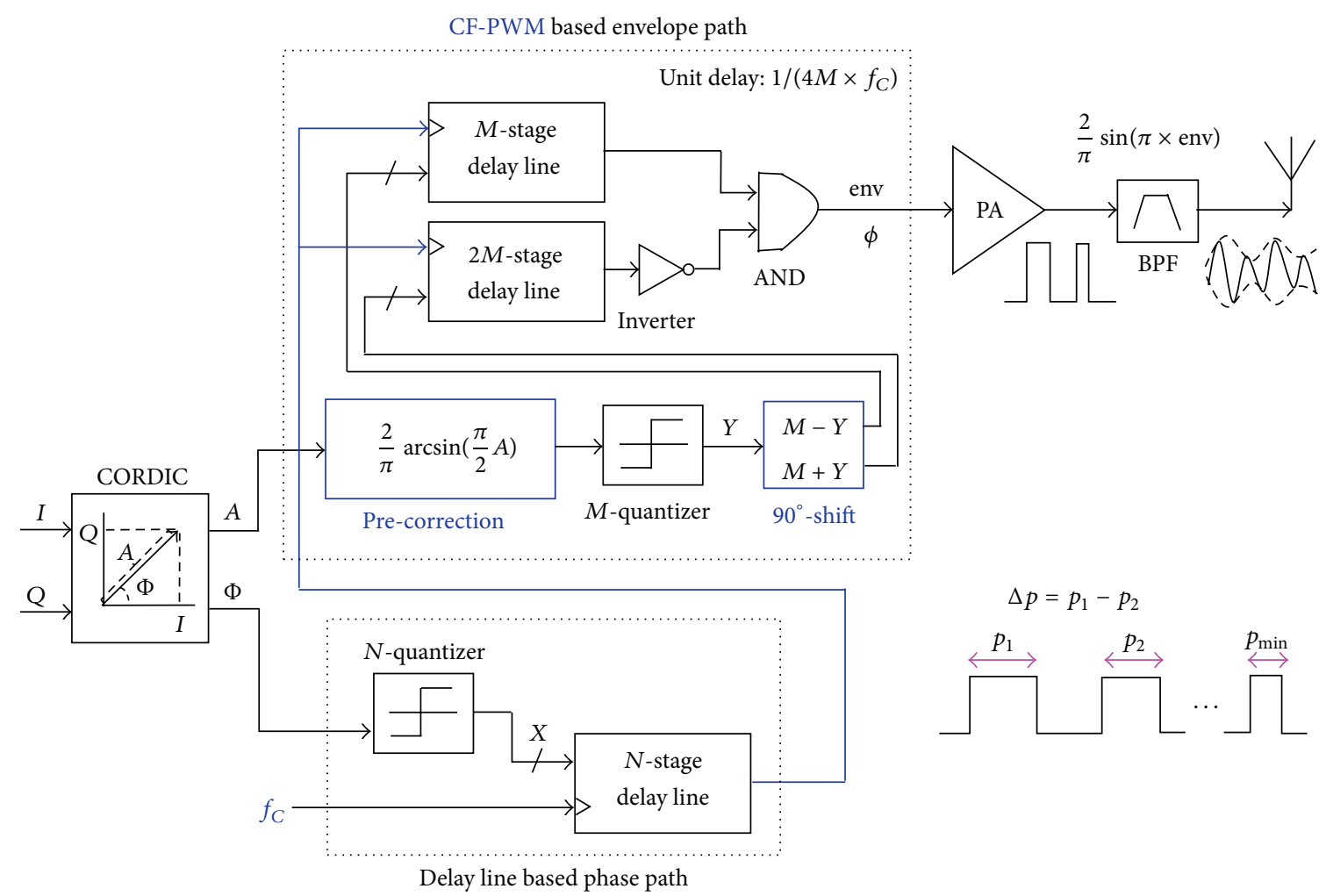

FIgURE 4: Proposed delay-line based CF-PWM mode for fully digital transmitter.

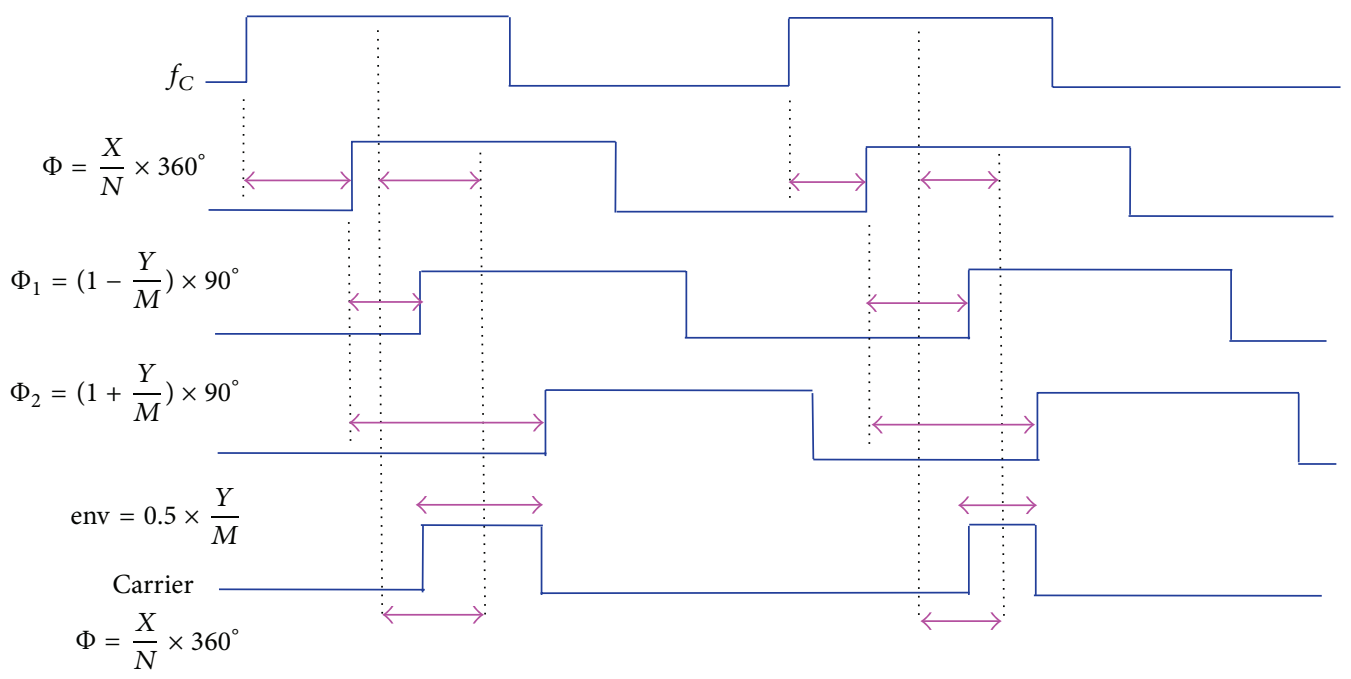

FIGURE 5: Function diagram of proposed CF-PWM architecture.

3.2. Proposed CF-PWM Mode. Figure 4 shows the proposed delay-line based CF-PWM mode for fully digital transmitter. Multibit phase components are mapped to the rising-edge positions of the carrier clock with a fixed duty cycle of 0.5 by employing an $N$-level quantizer and an $N$-stage delay line. Multibit envelope components are converted to the pulse widths of the position-modulated carrier clock through the delay differences between the $M$ - and $2 M$-stage delay lines and subsequent logic operations. Unlike IF-PWM mode, it is pulse widths rather than densities of the carrier clock that represent baseband envelopes. For ensuring the modulation linearity, both envelope precorrection module with antitrigonometric function and $90^{\circ}$ phase-shifted operation are added to compensate for the distortions caused by the BPF with variable pulse-width inputs, as discussed above.

The function diagram of the proposed CF-PWM architecture is shown in Figure 5. For a certain carrier clock, the $N$-stage delay line with a unit delay of $1 /\left(N \times f_{C}\right)$ converts 


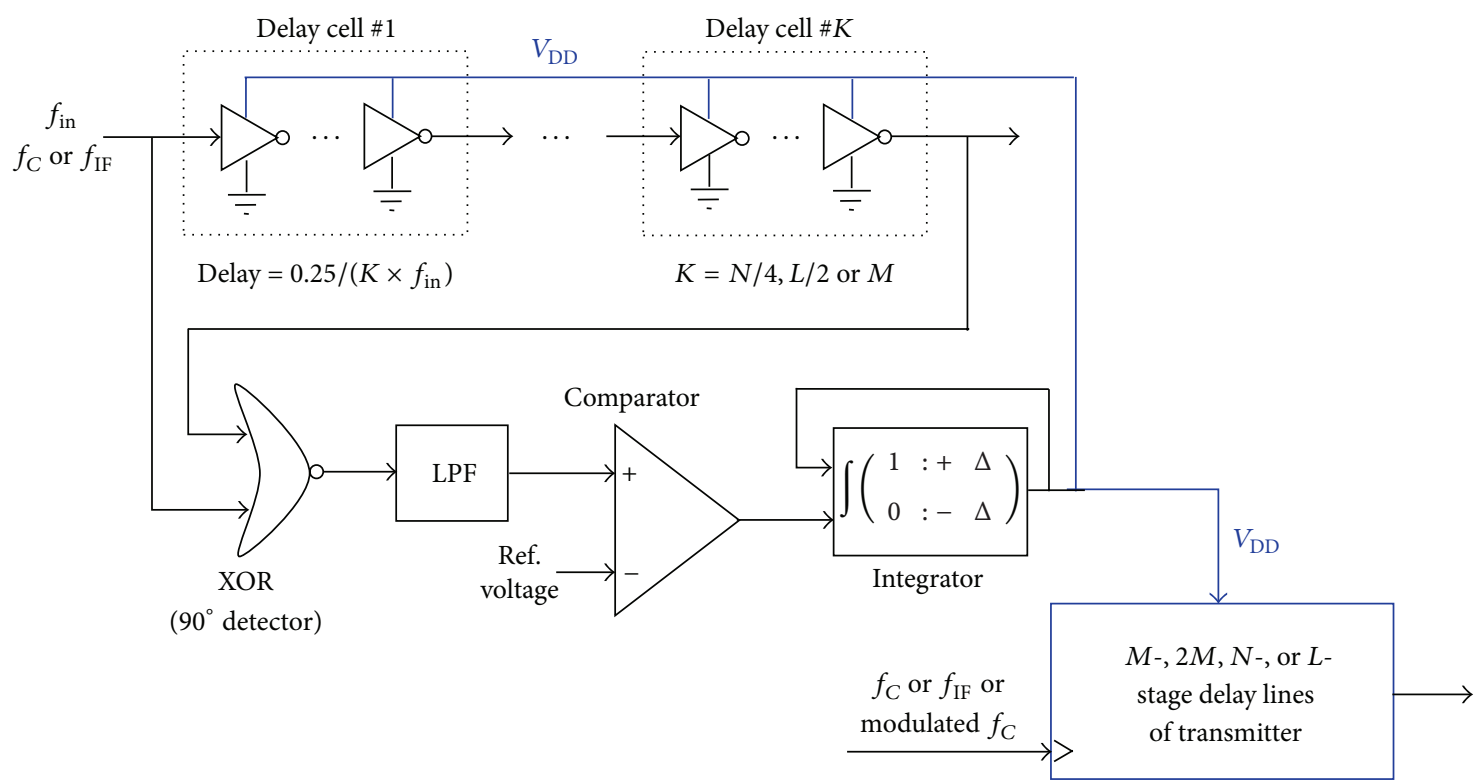

FIGURE 6: Proposed unit-delay autocalibration loop with reconfigurable $f_{C}$.

the quantized phase $X$ to the rising-edge lag of the carrier clock. The $M$ - and $2 M$-stage delay lines with a unit delay of $1 /\left(4 M \times f_{C}\right)$ subsequently map the precorrected quantization envelope $Y$ into two different rising-edge lags $\Phi_{1}$ and $\Phi_{2}$ on the symmetry of $90^{\circ}$ phase-shifted points. The pulse-width env generated by $\Phi_{1-2}$ represents the precorrected envelope component, and the lag $\Phi$ of the $90^{\circ}$ phase-shifted points reflects the phase component, respectively. That is, both pulse-widths and 1/4-period positions of the carrier clock convey baseband signals. Similarly, quantization resolutions or levels determine linearity. The modulated carrier clock $c(t)$ is shown in the following equation:

$$
\begin{aligned}
c(t)= & 0.5 \times \frac{Y}{M} \times \text { square }\left[\omega_{c} t-90^{\circ}+\Phi(t)\right] \\
= & 0.5 \times\left[\frac{2}{\pi} \arcsin \left(\frac{\pi}{2} \times \frac{A(t)}{A_{\text {std }}}\right)\right] \\
& \times \text { square }\left[\omega_{c} t-90^{\circ}+\Phi(t)\right] .
\end{aligned}
$$

The modulated sinusoidal carrier $y(t)$ is shown in (6). Continuous baseband envelope $A$ and phase $\Phi$ are transmitted. Consider

$$
\begin{aligned}
y(t)= & \frac{2}{\pi} \times \sin \left[\pi \times\left(0.5 \times\left[\frac{2}{\pi} \arcsin \left(\frac{\pi}{2} \times \frac{A(t)}{A_{\mathrm{std}}}\right)\right]\right)\right] \\
& \times \sin \left(\omega_{c} t-90^{\circ}+\Phi(t)\right) \\
= & \frac{1}{A_{\mathrm{std}}}\left[A(t) \times \sin \left(\omega_{c} t-90^{\circ}+\Phi(t)\right)\right] .
\end{aligned}
$$

The minimum pulse width $p_{\min }$ of the modulated carrier clock, shown in (7), is determined by the maximum CF $f_{C \text {, max }}$ and the minimum normalized envelope $A_{\text {std,min }}$.
The pulse width resolution $\Delta p$, shown in (8), depends on $f_{C}$ and envelope quantization level $M$ and needs to be recognized by the sequent $\mathrm{PA}$. Clearly, the ability of the PA conducting narrow pulses is a critical parameter for the proposed implementation. With finite signal rise and fall time, ultra narrow pulses going through the class-D PA are prone to be trapezoidal or triangular rather than being square, considering the limited working frequency or conducting capability of the present PA. That is why the proposed CFPWM architecture only aims for low CF range of $2 \mathrm{M}-$ $100 \mathrm{MHz}$. Consider the following equations:

$$
\begin{gathered}
p_{\min }=\frac{0.5}{f_{C, \max }} A_{\text {std, } \min }, \\
\Delta p=\frac{0.5}{M \times f_{C}} .
\end{gathered}
$$

3.3. Unit-Delay Autocalibration. To ensure modulation performances, the unit delay of delay lines needs to be accurate. To meet wide $f_{C}$ range, the unit delay also needs to be reconfigurable. In addition to $\mathrm{D}$ flip-flops apt for large unit delay, in most cases the unit delay is small and is implemented by inverters without or with resistive interpolation $[5,11]$. However, inverters require accurate delay calibration for high robustness over process and temperature variations. Figure 6 shows the proposed unit-delay calibration loop with wide and reconfigurable $f_{C}$.

The phase difference between the periodic clock input and $90^{\circ}$-shifted clock corresponding to $K$ unit delays is measured and converted to an error voltage by an XOR gate followed by a low-pass filter (LPF) and a sequent comparator. The error voltage sent to an integrator with driver buffer is accumulated and fed to the power supply $V_{\mathrm{DD}}$ of delay cells, which inversely modifies the unit delay. Under the negative feedback operation with closed-loop $V_{\mathrm{DD}}$ calibration, for 


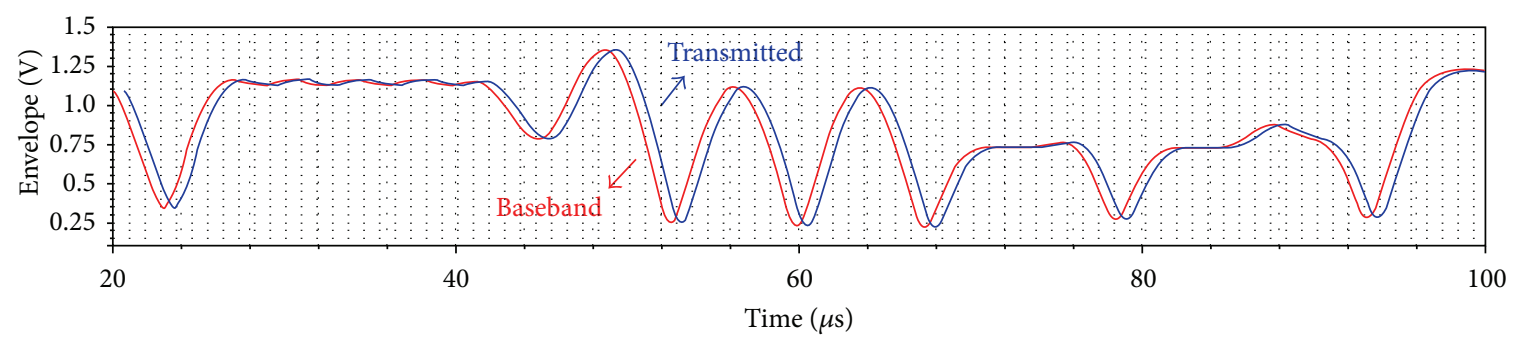

(a)

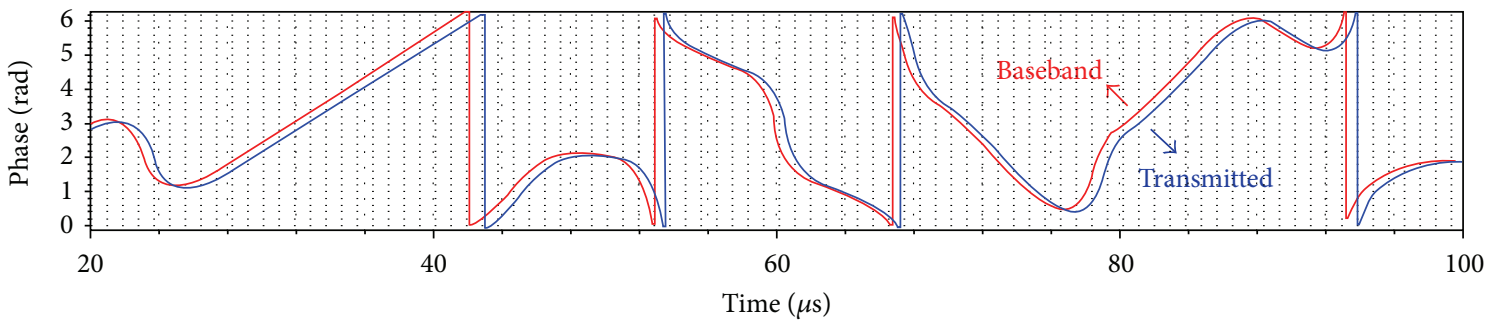

(b)

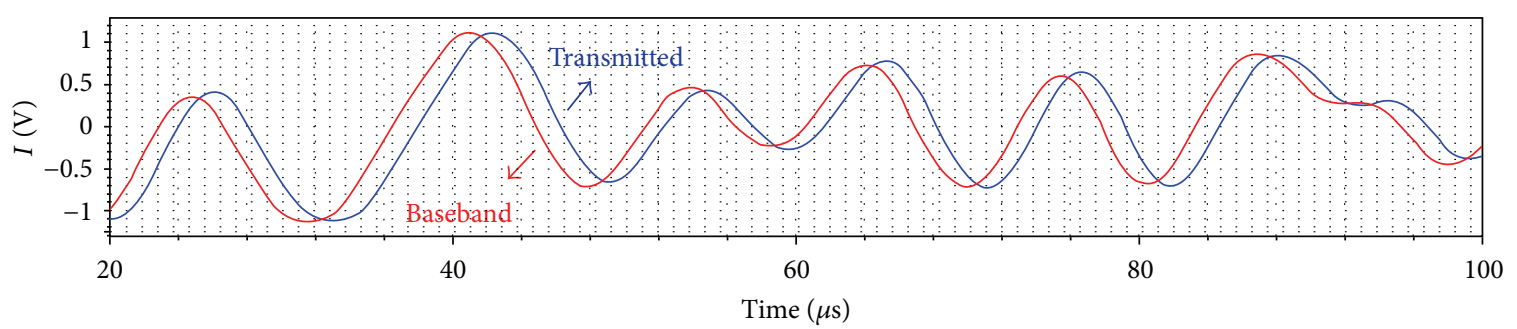

(c)

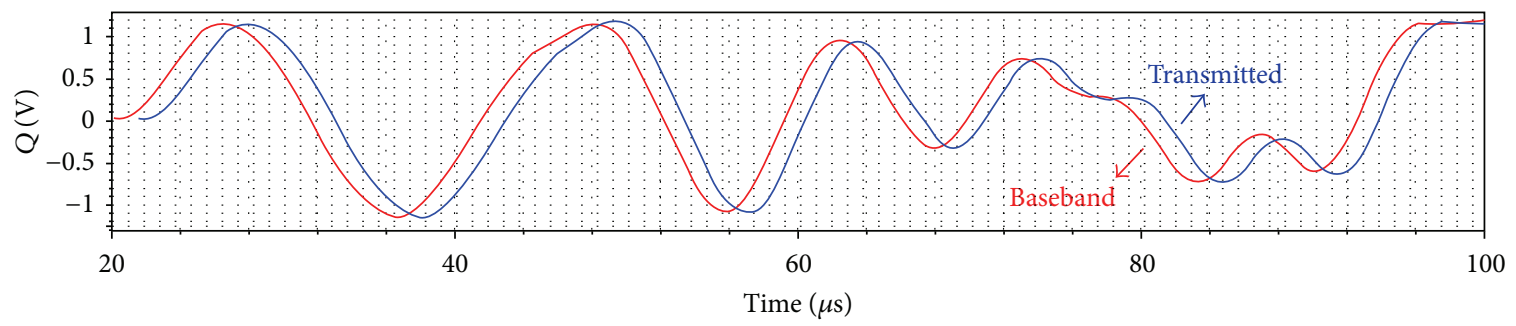

(d)

Figure 7: Simulated transmitter components conveyed by proposed architecture with comparison to baseband ones.

a certain clock $f_{\text {in }}\left(f_{\mathrm{C}}\right.$ or $\left.f_{\mathrm{IF}}\right)$, the unit delays of open-loop delay lines are accurately achieved by strict match designs and conform to (9), respectively, for different delay lines of the phase path and IF- and CF-PWMs. By setting matched inverter number in each delay cell and perfect $V_{\mathrm{DD}}$ range of delay cells, reconfigurable $f_{C}$ and corresponding unit delays are accomplished as follows:

$$
\begin{aligned}
\tau & =\frac{0.25}{K \times f_{\text {in }}} \\
& = \begin{cases}\frac{1}{N \times f_{C}}, & \text { Phase-Path }\left(K=\frac{N}{4}, f_{\text {in }}=f_{C}\right), \\
\frac{1}{2 L \times f_{\mathrm{IF}}}, & \text { IF-PWM }\left(K=\frac{L}{2}, f_{\text {in }}=f_{\mathrm{IF}}\right), \\
\frac{1}{4 M \times f_{C}}, & \text { CF-PWM }\left(K=M, f_{\text {in }}=f_{C}\right) .\end{cases}
\end{aligned}
$$

\section{Experimental Results}

The proposed fully digital transmitter is designed in $180 \mathrm{~nm}$ CMOS with external class-D PA and BPF. Excluding the PA, the proposed implementation only dissipates ultra low power of $100 \mu \mathrm{W}$ from $1.8 \mathrm{~V}$ power supply. The transmitter achieves a power efficiency of $70 \%$, according to circuit-level simulations. The quantization levels are 256 and 128 for the phase and envelope paths, respectively.

The supply voltage of delay cells can be changed from 1.4 to $2.2 \mathrm{~V}$, which corresponds to the minimum unit delays of 7.8-3.9 ps for the phase path, 120.3-60.1 ps for the IF-PWM, and 39.0-19.5 ps for the CF-PWM, according to circuit-level simulations of three groups of different delay lines in the nominal case. As a result, the CF ranges of $50-100 \mathrm{MHz}$ for the CF-PWM mode and $0.5-1 \mathrm{GHz}$ for the IF-PWM one are 

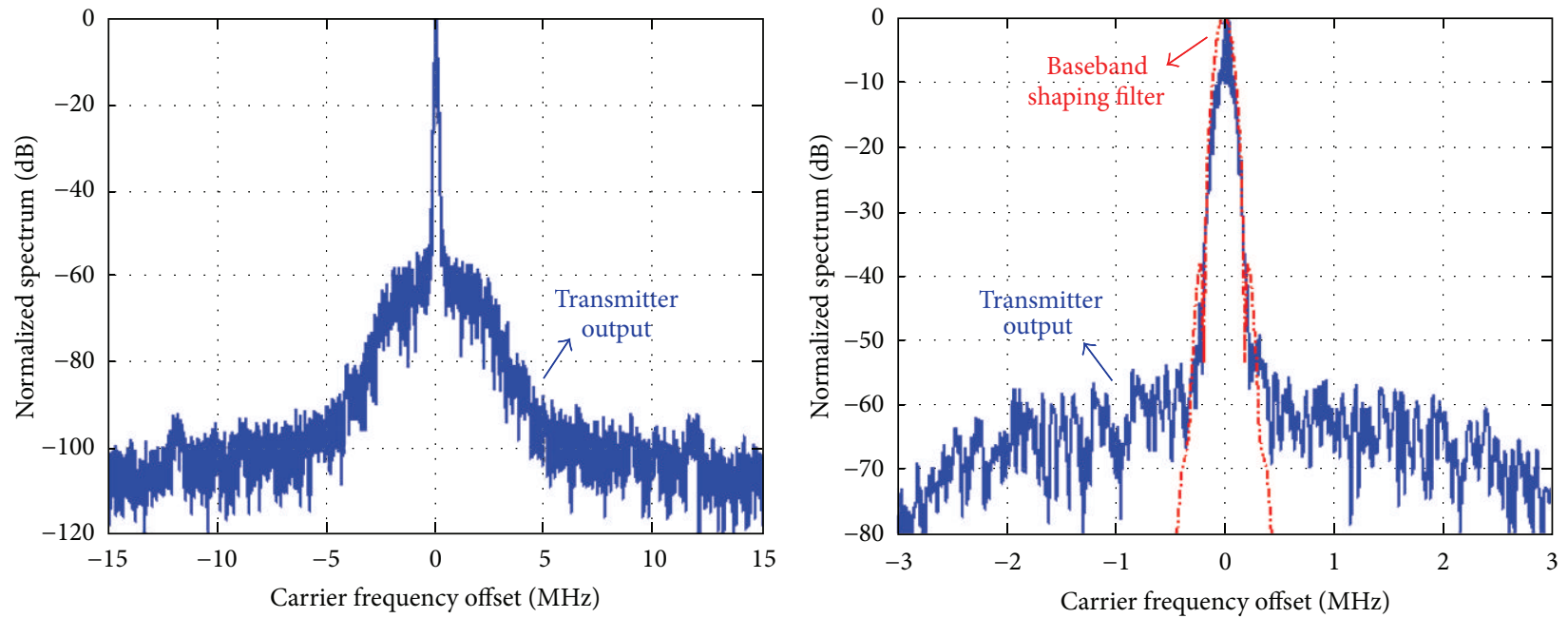

(a)
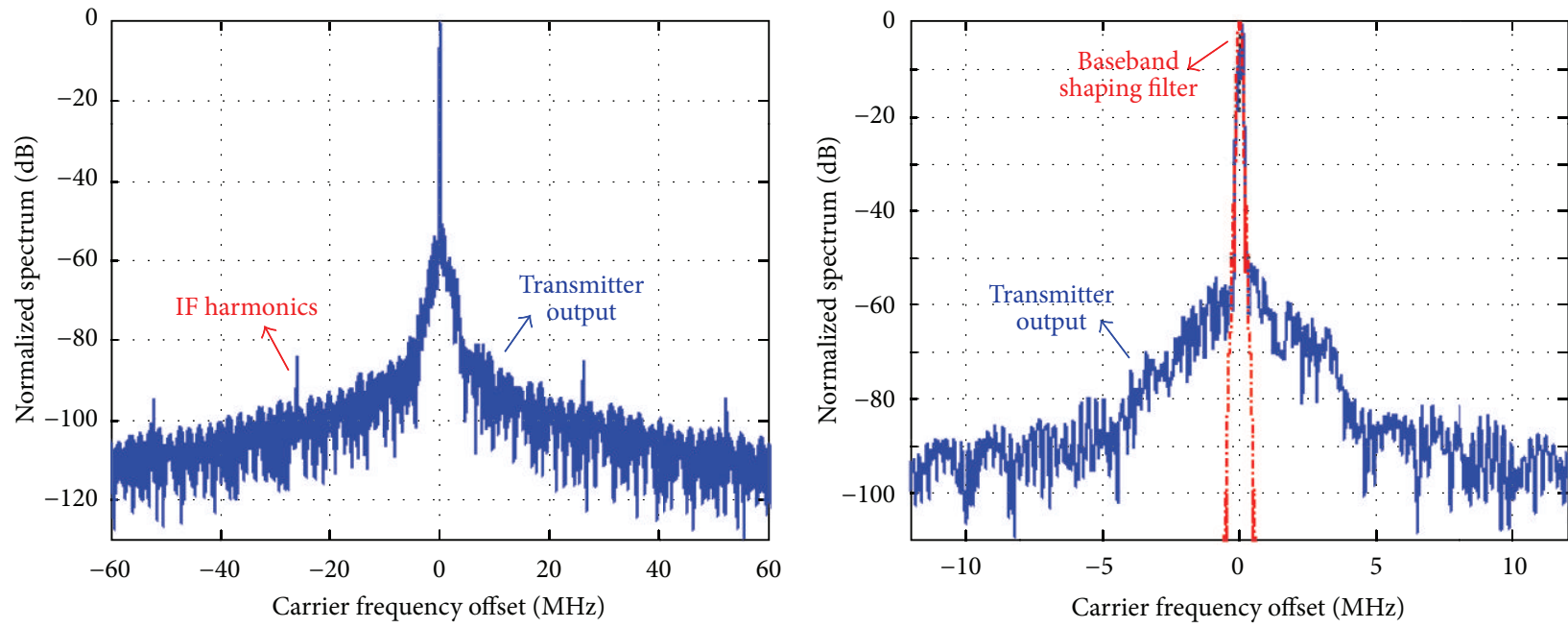

(b)

FIGURE 8: Simulated transmitter normalized far and near spectrums: (a) CF-PWM centered at 50 MHz; and (b) IF-PWM centered at 200 MHz.

gotten. By adding matched inverters in each group of delay cells, the lower CF range is also covered and the wide CF range of $2 \mathrm{M}-1 \mathrm{GHz}$ is achieved. For a certain baseband signal with normalized envelope range of $0.15-1.0$, with $f_{C}$ less than $100 \mathrm{MHz}$, the $p_{\min }$ and $\Delta p$ of the CF-PWM mode are $0.75 \mathrm{~ns}$ and 0.04 ns, respectively, which could be recognized by the present PA.

Figure 7 shows the simulated transmitter components conveyed by the proposed architecture with comparison to the baseband ones for 8PSK signal with an envelope range of $0.22 \sim 1.45 \mathrm{~V}$. The proposed transmitter conveys the baseband components very well with the simulated error vector magnitude (EVM) of 3\%. Little distortions result from limited quantization resolutions and slight PA push-pull asymmetry. An ideal receiver reconstructing the transmitted components is implemented in Matlab.

Figure 8 shows the simulated transmitter normalized far and near spectrums centered at $50 \mathrm{MHz}$ for CF-PWM mode and at $200 \mathrm{MHz}$ for IF-PWM one. The roll-off feature of spectrum side lobes is determined by the baseband shaping filter. The noise floor less than $60 \mathrm{~dB}$ is observed below the carrier, and $20 \mathrm{~dB}$ spectrum optimization is achieved when compared to the existing designs $[4,5]$ with the noise floor at $40 \mathrm{~dB}$ below the carrier. For CF-PWM mode, no any other frequency components including spurs and harmonics exist, except for the carrier. For IF-PWM mode, IF spurs located at even multiples of $f_{\mathrm{IF}}$ from $f_{\mathrm{C}}$ are observed and less than $-80 \mathrm{~dB}$. Hence, the proposed transmitter achieves high spectrum purity.

\section{Conclusions}

By combining delay-line based IF-PWM for high $f_{C}$ band and proposed CF-PWM with precorrection for low $f_{C}$ band, a fully digital transmitter is implemented in $180 \mathrm{~nm}$ CMOS, with wide CF range of $2 \mathrm{M}-1 \mathrm{GHz}$ and high power efficiency of $70 \%$. A unit-delay autocalibration circuit with closed-loop 
detection and open-loop adjustment is presented to support $f_{C}$ reconfiguration. The experimental results show that the proposed architecture transmits baseband components well with high spectrum purity and low EVM of 3\%. Compared to the existing designs, $20 \mathrm{~dB}$ spectrum optimization is achieved. Chip prototype will be considered as a future work to further verify the proposed transmitter architecture.

\section{Acknowledgments}

The authors would like to thank the National Natural Science Foundation of China (no. 61306037 and no. 61201182) for the financial support.

\section{References}

[1] J. Chen, L. Rong, F. Jonsson, G. Yang, and L.-R. Zheng, “The design of all-digital polar transmitter based on ADPLL and phase synchronized modulator," IEEE Journal of Solid-State Circuits, vol. 47, no. 5, pp. 1154-1164, 2012.

[2] H. Zhang and B. Chi, "All digital wideband polar transmitter," in Proceedings of the 2nd International Conference on Consumer Electronics, Communications and Networks (CECNet '12), pp. 1618-1621, April 2012.

[3] Henri Ruotsalainen et al., "A new quadrature PWM modulator with tunable center frequency for digital RF transmitters," IEEE Transactions on Circuits and Systems-II: Express Briefs, vol. 59, pp. 756-760, 2012.

[4] P. Midya, P. Wagh, and P. Rakers, "Quadrature integral noise shaping for generation of modulated RF signals," in Proceedings of the 45th Midwest Symposium on Circuits and Systems, pp. 537540, August 2002.

[5] P. A. J. Nuyts, P. Singerl, F. Dielacher, P. Reynaert, and W. Dehaene, "A fully digital delay line based $\mathrm{GHz}$ range multimode transmitter front-end in $65 \mathrm{~nm}$ CMOS," IEEE Journal of SolidState Circuits, vol. 47, pp. 1681-1692, 2012.

[6] P. A. J. Nuyts, B. Francois, W. Dehaene, and P. Reynaert, "A CMOS burst-mode transmitter with watt-level RF PA and flexible fully digital front-end," IEEE Transactions on Circuits and Systems-II: Express Briefs, vol. 59, pp. 613-617, 2012.

[7] M. Nielsen and T. Larsen, "An RF pulse width modulator for switch-mode power amplification of varying envelope signals," in Proceedings of the Topical Meeting on Silicon Monolithic Integrated Circuits in RF Systems (SiRF '07), pp. 277-280, January 2007.

[8] E. Lopelli, J. D. van der Tang, and A. H. M. van Roermund, "Minimum power-consumption estimation in ROM-based DDFS for frequency-hopping ultralow-power transmitters," IEEE Transactions on Circuits and Systems I: Regular Papers, vol. 56, no. 1, pp. 256-267, 2009.

[9] K. -W. Kim, A fully-integrated all-digital outphasing transmitter for wireless communications, [PHD dissertation], Georgia Institute of Technology, Atlanta, Ga, USA, 2009.

[10] A. Chen and S. Yang, "Reduced complexity CORDIC demodulator implementation for D-AMPS and digital IF-sampled receiver," in Proceedings of the IEEE GLOBECOM-The Bridge to the Global Integration, pp. 1491-1496, November 1998.
[11] S. Henzler, S. Koeppe, D. Lorenz, W. Kamp, R. Kuenemund, and D. Schmitt-Landsiedel, "Variation tolerant high resolution and low latency time-to-digital converter," in Proceedings of the 33rd European Solid-State Circuits Conference (ESSCIRC '07), pp. 194-197, September 2007. 

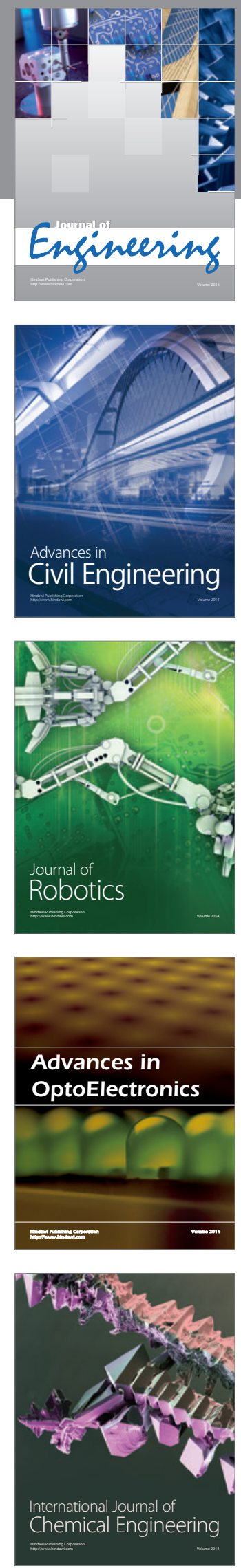

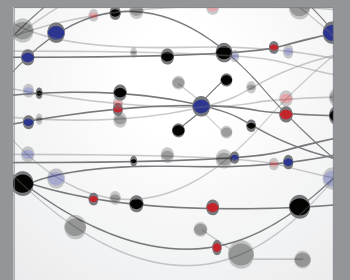

The Scientific World Journal
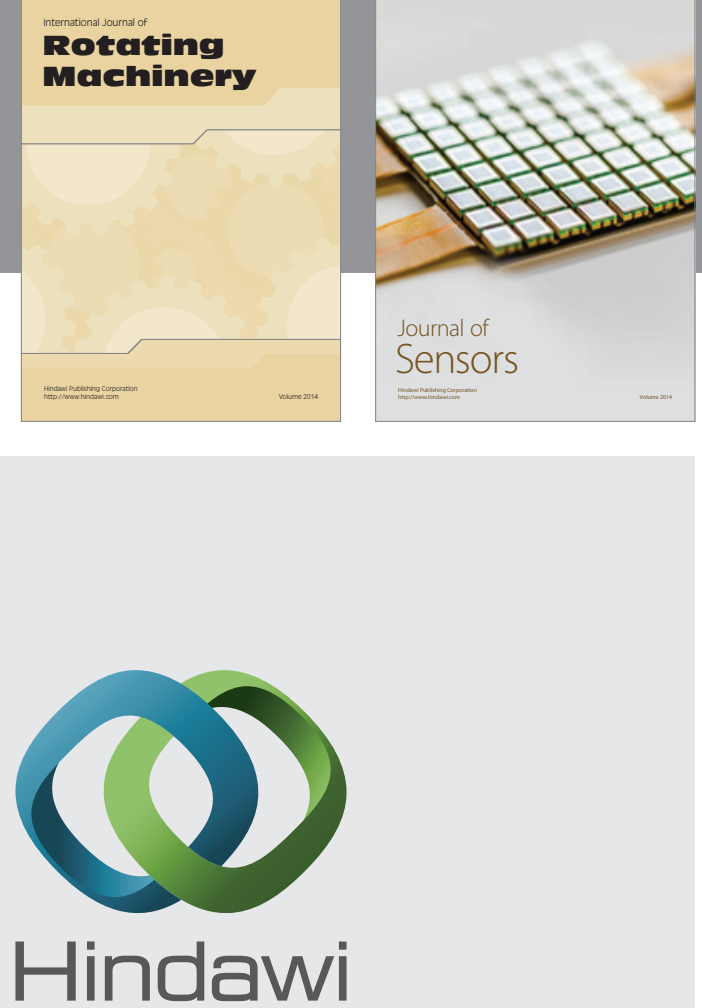

Submit your manuscripts at http://www.hindawi.com
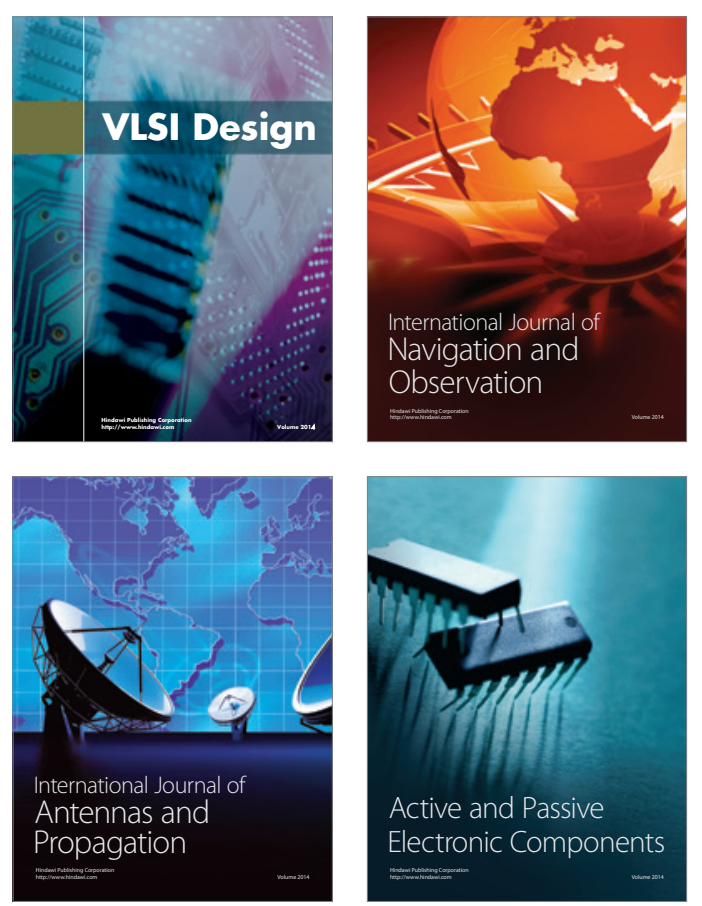
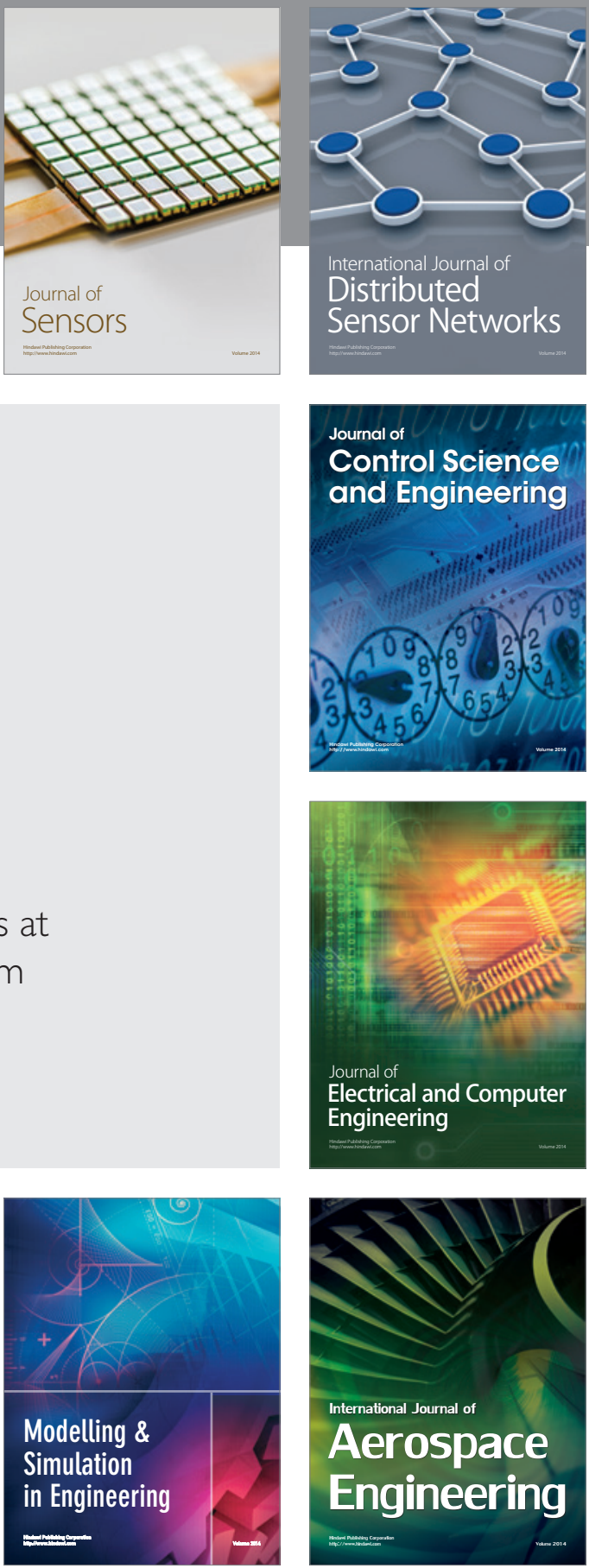

Journal of

Control Science

and Engineering
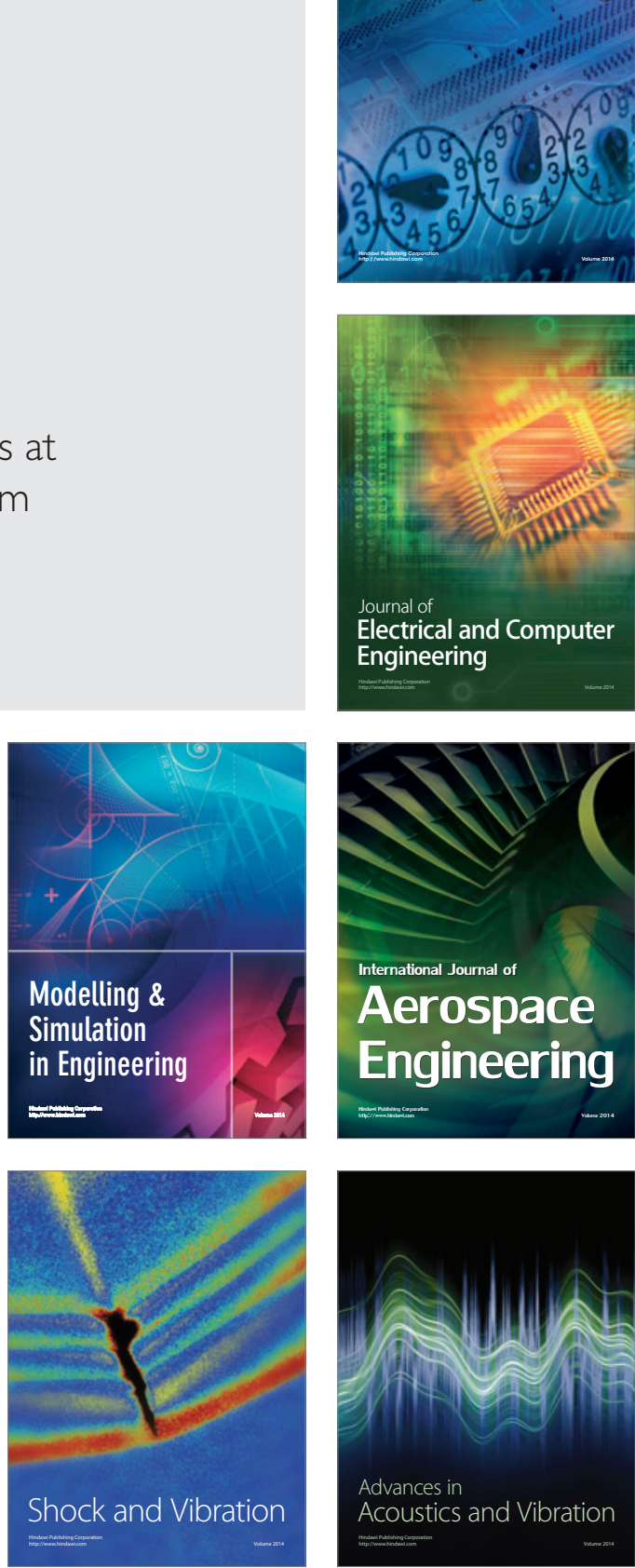